\title{
Operation optimization of multi-energy flow integrated energy system based on multi-objective
}

\author{
Yongli Wang ${ }^{1}$, Xin Chen ${ }^{1 *}$, Lisha Fan ${ }^{2}$, Minhan Zhou ${ }^{1}$, Huanran Dong ${ }^{1}$, Kunpeng Zhao ${ }^{3}$, Shaokun Jia ${ }^{4}$ \\ ${ }^{1}$ School of Economics and Management, North China Electric Power University, Beijing, 102206, China \\ ${ }^{2}$ State Grid Jibei Integrated Energy Service Co., Ltd, Beijing 100045 China \\ ${ }^{3}$ Office State Grid Corporation Customer Service Center Tianjin, 300309, China \\ ${ }^{4}$ Technical Support Center Beijing Fibrlink Communication CO., LTD. Tianjin Branch, Tianjin, 300380, China
}

\begin{abstract}
In order to solve the problem that most of the existing combined cooling, heating and power integrated energy systems only consider the investment cost of the system unit or the system environmental pollution affecting the overall optimization of the system, with the goal of system economy and environmental protection, the construction of gas turbines, gas boilers, The optimization model of the combined cooling, heating and power system of electric refrigerators and other units and the establishment of constraint conditions; according to the requirements of the cooling, heating, and electric loads in the dispatch cycle, as well as the energy supply efficiency of each equipment of the system, the starting and stopping power and other operating parameters are in the interval. Based on the respective data, a multi-energy flow regional integrated energy system economic dispatch optimization operation strategy optimized within the dispatch period is proposed. The results show that the operation strategy can take into account the economy and environmental protection of the system at the same time, make the system operation more optimized.
\end{abstract}

\section{Introduction}

Existing energy systems are often planned and operated separately, resulting in low energy utilization and high pollution [1]. Nowadays, people are more researching how to coordinate and optimize each independent energy supply system to reduce its environmental pollution while increasing energy efficiency and economic performance [2]. The large-scale access of various energy sources has led to the fact that the energy system is often unable to balance economy and environmental protection, and the ability to optimize operation is insufficient. Therefore, how to optimize the integrated energy system and take into account the economy and environmental protection of system operation has become a problem that needs to be solved [3]. Ref. [4] used linear models to configure the optimal capacity of power, natural gas, and thermal systems to improve the energy utilization efficiency of the system; Ref. [5] considered carbon emissions and the consumption of renewable energy, and proposed a new hybrid power flow calculation method to ensure the economic and stable operation of the combined heat and power microgrid.

$$
C_{C C H P}=R\left(C_{\mathrm{inf} . G T} P_{c a p . G T}+C_{\mathrm{inf} . G B} P_{c a p . G B}+C_{\text {inf.GE }} P_{c a p . G E}+C_{\text {inf.EC }} Q_{c a p . E C}\right)+C_{\text {inf.AC }} Q_{c a p . A C}
$$

In formula (2): $R$ is the capital recovery rate; $P_{\text {cap.GT }}, P_{\text {cap.GB }}, P_{\text {cap.GE }}$ are the rated electric power of gas turbines, gas boilers, and natural gas internal
The objective function is established with the optimal system economy and environmental protection, and the constraint conditions are established, and the optimization model of the combined cooling, heating and power (CCHP) system is established; finally, the results are analyzed and studied in combination with the calculation example. The system's operating results under a single goal and taking into account multiple goals at the same time provide a reference for the later collaborative optimization of IES.

\section{2 collaborative optimization objective function of IES}

The goal is to minimize the economic cost and optimal environmental protection of the integrated energy system. The economic cost of the system is mainly divided into unit investment cost $C_{C C H P}$, electricity purchase cost $C_{e}$ and natural gas purchase cost $C_{g}$. The objective function is

$$
C_{J}=C_{C C H P}+C_{e}+C_{g}
$$

combustion engines respectively; $Q_{\text {cap.EC }}$ and $Q_{\text {cap.AC }}$ are electric refrigerators, The rated cold power of

\footnotetext{
* Corresponding author: cx1998@ncepu.edu.cn
} 
absorption chillers; $C_{\text {inf.GT }}, C_{\text {inf.GB }}, C_{\text {inf.GE }}, C_{\text {inf.EC }}$, $C_{\text {inf.AC }}$ are gas turbines, gas boilers, natural gas internal combustion engines, electric refrigerators and absorption chillers within the rated power, respectively operating costs.

$$
C_{e}=\sum_{t=1}^{T} c_{e, t} P_{e, t}
$$

In formula (3): $T$ is one operating cycle of the system; $C_{e}$ is the unit price of electricity purchased by the system during t period, and $P_{e}$ is the purchase power of the system during t period.

$$
C_{g}=\sum_{t=1}^{T} c_{g} P_{e, t}
$$

In formula (4): $C_{g}$ is the unit price of natural gas purchased by the system during the period t; $P_{e, t}$ is the gas purchased power of the system during the period.

During system operation, the combustion of natural gas produces carbon dioxide gas. Therefore, when the system is running, the emission: the objective function of the environmental protection $\operatorname{cost} \mathrm{C}_{\mathrm{H}}$ of carbon dioxide is

$$
C_{H}=W_{C O_{2}}\left[\varepsilon_{e} P_{E C}^{I N}+\varepsilon_{g}\left(P_{G T . g}+P_{G B . g}+P_{G E . g}\right)\right]
$$

In formula (5): $W_{\mathrm{CO}_{2}}$ is the unit price of $\mathrm{CO}_{2}$ emission; $\varepsilon_{e}$ is the emission coefficient of carbon dioxide per unit of electric power; $\varepsilon_{g}$ is the carbon dioxide emission coefficient of unit volume of natural gas. The overall system optimization function $C_{Z}$ is

$$
C_{Z}=\min \left(C_{J}+C_{H}\right)
$$

\section{Integrated energy system modeling and constraints}

\subsection{Integrated energy system modeling and constraints}

The CCHP is the most commonly used in IES. The mathematical model and constraints of the gas turbine are

$\left\{\begin{array}{l}P_{G T . e}=\eta_{G T}^{e} P_{G T . g} \\ P_{G T . \mathrm{h}}={ }_{G T}^{h} P_{G T . g}\end{array}\right.$

Where: $P_{G T \text {.e }}$ is the power generation of gas turbine; $P_{G T \text {.h }}$ is the heat generation power; $\eta_{G T}^{e}$ is the power generation efficiency; $\eta_{G T}^{h}$ is the heat generation efficiency; $P_{G T \text {.g }}$ is the gas consumption power.

The mathematical model and constraints of the gas boiler are

$P_{G B . \mathrm{h}}=\eta_{G B}^{h} P_{G B . \mathrm{g}}(8)$

Where: $P_{G B \text {.h }}$ is the heating power of the gas boiler; PGB. $\mathrm{g}$ is the gas consumption power; $\eta_{G B}^{h}$ is the heat generation efficiency.

The mathematical model of a natural gas internal combustion engine is

$\left\{\begin{array}{l}P_{G E . \mathrm{e}}=\eta_{G E}^{e} P_{G E . \mathrm{g}} \\ P_{G E . \mathrm{h}}=\eta_{G E}^{h} P_{G E . \mathrm{g}}\end{array}\right.$

Where: $P_{G E \text {.e }}$ is the power generated by natural gas internal combustion engine; $P_{G E \text {.h }}$ is the heat generation power; $\eta_{G E}^{e}$ is the power generation efficiency; $\eta_{G E}^{h}$ is the heat generation efficiency; $P_{G E . \mathrm{g}}$ is gas consumption power. The mathematical model of the electric refrigerator is

$Q_{E C}^{O U T}=C O P_{E C} P_{E C}^{I N}(10)$

In formula (14): $C O P_{E C}$ is the output cooling power of the electric refrigerator; $P_{E C}^{I N}$ inputs the electric power; $C O P_{E C}$ is the cooling coefficient of the electric refrigerator.

The mathematical model of the absorption chiller is

$Q_{A C}=C O P_{A C} Q_{A C}^{h}(11)$

In formula (15): $Q_{A C}$ is the output cooling power of the absorption chiller; $C O P_{A C}$ is the cooling coefficient; $Q_{A C}^{h}$ is the input heating power.

The power balance constraint relation of electric energy, natural gas, heat energy and cold energy in this system is

$$
\left\{\begin{array}{l}
P_{e, t}+P_{G T . e}+P_{G E . e}-P_{E C}^{I N}=L_{e} \\
P_{G T . \mathrm{h}}+P_{G B . h}+P_{G E . \mathrm{h}}+Q_{A C}=L_{h} \\
P_{\mathrm{g}, t}+P_{G T . \mathrm{g}}+P_{G B . \mathrm{g}}+P_{G E . \mathrm{g}} Q_{A C}^{g}=L_{g} \\
P_{E C}^{O U T}+P_{A C}=L_{c}
\end{array}\right.
$$

Where: $L_{e}$ is the electrical load delivered by the system to the user side; $L_{h}$ is the heat load delivered by the 
system to the user side; $L_{g}$ is the gas load delivered by the system to the user side; $L_{c}$ is the cooling load delivered by the system to the user side.

\section{Case analysis}

In order to verify the economics of the regional integrated energy system dispatching strategy for cooling, heating and power, and the correctness of the established system model, the summer daily load data of an office building in a park is used as the load data of the system to carry out the system Optimal scheduling, using 0-1 mixed integer linear programming method to solve the dynamic economic model.

The rated capacity of each equipment in the calculation example system in this paper is as follows: (1)

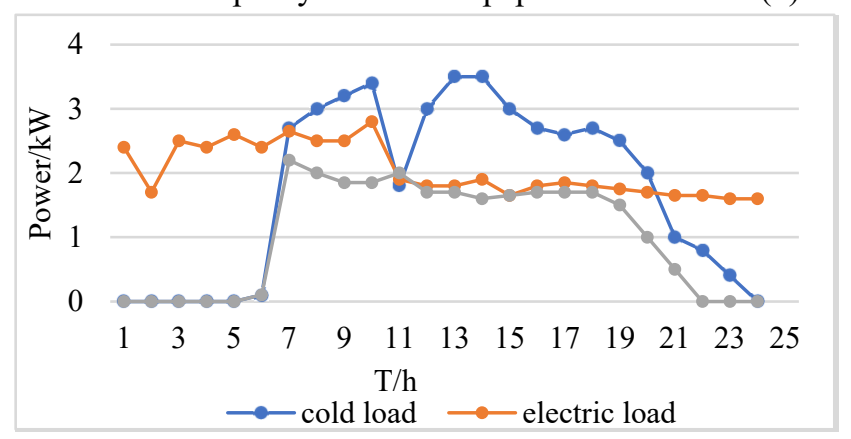

Figure1. Forecast value of cold, heat and electric load
The micro gas turbine is a micro gas turbine with a rated capacity of $200 \mathrm{~kW}$; (2) The heat exchanger rated power is $180 \mathrm{~kW}$; (3) The rated heating power of the electric boiler is $150 \mathrm{~kW}$; (4) The rated cooling capacity of the absorption chiller is $200 \mathrm{~kW}$; (5) The rated power of the electric chiller is $200 \mathrm{~kW}$.

The system includes a gas turbine generator, a natural gas internal combustion engine and a set of power storage equipment. The price of natural gas in the system is 3.12 yuan $/ \mathrm{m}^{3}$, and the daily load curves and electricity prices of various energy sources are shown in Figures 1 and 2. Based on the improved algorithm, the combined cooling, heating and power system model is optimized and solved with the best economic efficiency, the best environmental protection, and the comprehensive consideration of system economy and environmental protection. The solution results are shown in Table 1.

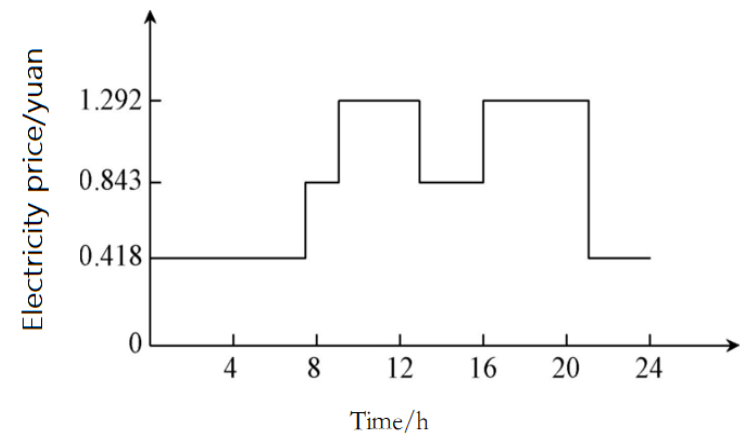

Figure 2. Electricity price chart

Table 1. Electricity price (Unit: ten thousand yuan)

\begin{tabular}{lccc}
\hline Optimization goal & $C_{J}$ & $C_{H}$ & $C_{Z}$ \\
\hline Minimal operating cost & 1103.23 & 60.32 & 1163.55 \\
Minimal environmental cost & 1131.63 & 57.04 & 1188.67 \\
Minimal overall cost & 1103.79 & 57.58 & 1161.47 \\
\hline
\end{tabular}

The system model fully meets the needs of cold, heat, and electricity loads. The specific conditions of the optimized scheduling operation are as follows:

1) During periods of low electricity prices, since the overall economic benefits of CCHP with micro-gas turbines are not as good as the economic benefits of purchasing electricity from the grid, energy is mainly supplied by the grid.

2) During the period of flat electricity price, the economic benefits of CCHP supply with micro-gas turbines are equivalent to the economic benefits of power grid purchase.

3) At peak power prices, the economic benefits of CCHP with micro-gas turbines are better than those of power grid purchases.

\section{Conclusion}

In this paper, an optimization model of CCHP is established, and the objective function and constraint conditions are constructed with the lowest operating cost and the lowest environmental cost caused by $\mathrm{CO}_{2}$ emissions. The model solution results prove that the improved solution can take into account the economic and environmental aspects of the system.

\section{Acknowledgement}

The authors would like to thank the support of the project "Science and Technology Foundation of State Grid Corporation of China (SGTJDK00DWJS1900272)".

\section{References}

1. Li Bing, Niu Honghai, Chen Jun, et al. (2018) Optimal operation of multi-energy collaboration system[J]. Distributed Energy, 11(2): 55-59

2. Liu M, Shi Y, Fang F. (2012) A new operation strategy for CCHP systems with hybrid chillers[J]. Applied Energy, 95( 2): 164-173 
3. Geidl M, Andersson G. (2005) A modeling and optimization approach for multiple energy carrier power flow[C]//Power Tech, 2005 IEEE Russia Power. New York: IEEE, 1-7.

4. Pei Wei, Deng Wei, Shen Ziqi, et al. (2014) Energy coordination and optimization of hybrid microgrid based on renewable energy and CHP supply[J]. Automation of Electric Power Systems, 38(16): 9-15.

5. Xu Xiandong, Jia Hongjie, Jin Xiaolong, et al. (2015) Study on hybrid heat-gas-power flow algorithm for integrated community energy system $[\mathrm{J}]$. Proceedings of the CSEE, 35(14): 3634-3642. 